\title{
Compte rendu
}

\section{Bessora. La Dynastie des boiteux. Tome 1: Zoonomia. Le Serpent à plumes. 2018, 423 p.}

\author{
Chris Reyns-Chikuma \\ University of Alberta
}

Bessora (un pseudonyme) est née à Bruxelles en 1968, d'une mère suisse (sans profession mentionnée) et d'un père gabonais, diplomate. Elle a habité dans de nombreux pays, dont les É.-U., la France, la Suisse, et plusieurs pays en Afrique. Elle a fait des études de finances puis d'anthropologie. Elle a publié plus de 10 romans en 20 ans, dont le premier en 1999, intitulé $53 \mathrm{~cm}$. Elle a aussi écrit son premier scénario pour une bande dessinée intitulée Alpha: De Abidjan à la Gare du Nord qui raconte le voyage d'un jeune Africain de Côte d'Ivoire qui cherche à rejoindre sa femme et son enfant qui habiteraient à Paris près de la Gare du Nord (qui lui sert d'Étoile du Nord), et qui a reçu de nombreux prix (dont le Pen Award) et a été traduite récemment en anglais et publiée chez The Bucket List.

Zoonomia est le premier volume d'une tétralogie, intitulée La Dynastie des boiteux, dont le deuxième volume vient de paraitre fin octobre 2018 (Citizen Narcisse). Ce roman de 400 pages raconte l'histoire sociale du $19^{\mathrm{e}}$ siècle sous un angle peu connu à travers l'arrivée d'un «bâtard » (Johann) comme il y en a eu des millions, de mères «indigènes » et de père blanc colonisateur. Il arrive à Paris, au départ avec le même type d'ambition que son père, à savoir être explorateur, et après être devenu apprenti taxidermiste, part à l'aventure en Afrique et aux É.-U.. Son obsession est d'être le premier «blanc» à tuer un gorille. Cette fiction est inspirée d'un vrai personnage (Paul Belloni du Chaillu) et permet de raconter la société française de ce $19^{\mathrm{e}}$ siècle déjà bien fictionnalisé et connu à travers les nombreux textes, classiques, comme Balzac (pour concurrencer l'état civil des Blancs) et Dumas (quarteron). Ces deux auteurs sont régulièrement cités dans le récit avec d'autres textes, soit explicitement comme De la littérature des nègres (1808) de l'Abbé Grégoire, soit plus implicitement comme le petit "Chimney Sweeper » (" un garçonnet noirci de charbon », p. 90), soit encore à travers une littérature aujourd'hui beaucoup moins connue, des récits d'aventures aux récits d'explorations (tout autant fictionnels) et aux premiers récits anthropologiques (souvent ambivalents et problématiques sous leurs prétentions scientifiques). 
Les allusions à Balzac, surtout à travers la Femme de 30 ans que lit l'épouse officielle de son père dans le récit, sont là pour s'y opposer dans la mesure où La Comédie bumaine décrit la société française de cette époque de manière supposément encyclopédique, mais ne le faisant que partiellement et partialement. En effet, Balzac ne fait que très peu référence, par exemple, au colonialisme pourtant déjà bien présent dans la société française et européenne. Bessora, au contraire, décrit un Paris et une France où les explorateurs, les colonisateurs, et leurs trophées coloniaux ("achetés », malacquis, volés...) qui enrichissent les collections comme le musée Perrin renforce tout le système de pensée classificatoire et cette obsession des hiérarchies des d'animaux et des races humaines. Dumas est évidemment le quarteron qui à l'époque a dû faire face au racisme sans trop aborder le problème dans ses œuvres (sauf dans George), et qui de suite après sa mort a été enseigné comme sans (trop) mentionner son héritage.

Le roman de Bessora est aussi un texte féministe dénonçant la hiérarchie dans laquelle la femme/les femmes est/sont toujours au bas tant chez les Blancs européens que chez les Noirs-Africains (p. 325). Ceci est encore métaphorisé par son obsession de «tuer ces animales [sic] » (326) et d'être le premier à rencontrer un(e) gorille.

La langue de Bessora est d'ailleurs intéressante. Elle est d'abord faite d'un riche vocabulaire (de l'ordinaire comme «glaviot» ou technique pour la taxidermie ou l'Afrique), et d'expressions idiomatiques. Le roman utilise aussi la $2^{\mathrm{e}}$ personne, une technique assez rare, puisque l'on en compte une dizaine d'occurrences en littérature française. Soit dans le «tu» dont la première occurrence française systématique remonterait à 1918 (Le Serviteur d'Henri Bachelin aujourd'hui totalement oublié) et des romans d'avant-garde comme Perec et Calvino, soit le "vous » habituellement associé au roman d'avantgarde (p. ex., La Modification de M. Butor, 1957). Aucun récit francophone sur l'Afrique n'a utilisé ces $2^{\mathrm{e}}$ personnes, «tu» ou «vous». Ce «tu» a ici pour effet de renforcer l'identification entre le lecteur/trice blanc qui représente la majorité des acheteurs de ce livre et le protagoniste, qui, vu sa condition physique (boîteux) et raciale, aurait autrement pu l'en distancer, mais sans doute davantage de l'interpeller. Enfin, l'usage du passé simple et de subjonctifs passés renforce une atmosphère fictionnelle et spécialement celle des romans du $19^{\mathrm{e}}$ siècle, mais ici avec une certaine ironie. Les descriptions baroques et burlesques de personnages historiques comme l'arrière-arrière-ettrès-arrière-petit-fils de Myles Standish (un «pilgrim » de la colonie de Plymouth) comme pétomane, sénile, fou qui tire un coup de fusil pour appeler sa bonne au lieu de faire tinter la sonnette qui est là pour cela, comme un être immonde d'arrogance de race, de caste, et de classe tout à la fois (p. 248), donnent une dimension mythique au roman qui l'insère aussi dans le genre 
roman d'aventure, ce qu'il est aussi. Tout cela reflète l'ambiance «classifiraciste » qui régnait en France et en Europe au $19^{\mathrm{e}}$ siècle, du grand-père de Darwin qui créa le terme de «zoonomia » dans son traité de 1792, à 1848, date de la fin de l'esclavage par Victor Schœlcher, et au-delà, parallèle et inextricablement mêlée à la recherche scientifique et aux luttes sociales. Ce roman est un contrepoint superbe et nécessaire à Balzac et Dumas. Il est aussi très agréable à lire grâce aussi à son humour et son ironie. 